\title{
Dynamic Range of Mass Accuracy in LTQ Orbitrap Hybrid Mass Spectrometer
}

\author{
Alexander Makarov, Eduard Denisov, Oliver Lange, \\ and Stevan Horning \\ Thermo Electron (Bremen) GmbH, Bremen, Germany
}

\begin{abstract}
Using a novel orbitrap mass spectrometer, the authors investigate the dynamic range over which accurate masses can be determined (extent of mass accuracy) for short duration experiments typical for LC/MS. A linear ion trap is used to selectively fill an intermediate ion storage device (C-trap) with ions of interest, following which the ensemble of ions is injected into an orbitrap mass analyzer and analyzed using image current detection and fast Fourier transformation. Using this technique, it is possible to generate ion populations with intraspectrum intensity ranges up to $10^{4}$. All measurements (including ion accumulation and image current detection) were performed in less than $1 \mathrm{~s}$ at a resolving power of 30,000. It was shown that 5-ppm mass accuracy of the orbitrap mass analyzer is reached with $>95 \%$ probability at a dynamic range of more than 5000, which is at least an order of magnitude higher than typical values for time-of-flight instruments. Due to the high resolving power of the orbitrap, accurate mass of an ion could be determined when the signal was reliably distinguished from noise $\left(\mathrm{S} / \mathrm{N}_{p-p}>2 \ldots .3\right)$. (J Am Soc Mass Spectrom 2006, 17, 977-982) (C) 2006 American Society for Mass Spectrometry
\end{abstract}

$\mathrm{T}$ The dynamic range over which accurate measurements of mass can be made ("extent of mass accuracy") is a key analytical figure-of-merit for any accurate-mass analyzer. In practice, such analyzers are coupled to liquid chromatography or other separation methods, and measurements are made for transient signals (e.g., with spectra recorded at a rate of 1 spectrum/s). For any analyzer, mass accuracy is limited statistically by too few ions detected or by peak position shifts due to too many ions. Though being a universal problem, limitations to the extent of mass accuracy have been investigated in detail for time-of-flight (TOF) mass analyzers. These analyzers are particularly susceptible to variations in ion intensity because they use fast acquisition systems with inherently modest dynamic range [1-4]. Even with lock-mass and analyte signals far from saturation, a strong dependence of peak position on peak intensity is observed. As a result, $5 \mathrm{ppm}$ r.m.s. mass accuracy cannot be achieved over a signal range larger than a few hundred in $1 \mathrm{~s}$ acquisition in ion counting TOFs, even when advanced algorithms for intensity correction are employed [1-3]. TOFs with analog detection are in principle capable of a dynamic range of around one thousand [4].

In Fourier transform ion cyclotron resonance (FT ICR) mass spectrometry, mass accuracy at low signal intensities is limited by the imprecision of peak cen-

Published online June 5, 2006

Address reprint requests to Dr. A. A. Makarov, Thermo Electron (Bremen) GmbH, Hanna Kunath Strasse 11, Bremen 28199, Germany. E-mail: alexander.makarov@thermo.com troiding introduced by the noise of the image current preamplifier [5-8]. Unlike TOFs, FT ICR employs much slower acquisition systems with much higher dynamic range. At high intensities, Coulomb repulsion, rather than detector saturation, produces mass shifts that depend not only on the total charge [9-11] but also on the intensities of individual mass peaks $[12,13]$. Overall, intrascan dynamic range of a few thousand is possible $[8,14]$ with mass accuracy of a few ppm.

This work investigates the extent of mass accuracy for a novel Fourier transform mass spectrometer: LTQ Orbitrap. This instrument combines a linear ion trap with radial ejection [15] and an orbitrap mass analyzer [16]. The orbitrap mass analyzer is an electrostatic trap wherein tangentially injected ions rotate around a central electrode, being confined by applying an appropriate voltage between the outer and central electrodes. Mass analysis is based on image current detection of frequencies of axial oscillations. Therefore, its extent of mass accuracy is limited by the same factors as FT ICR. The objective of this work is to determine upper and lower limits of ion intensities for accurate mass analysis and ways for their improvement.

\section{Experimental}

All experiments were carried out using a mixture of Ultramark 1600 (Lancaster Synthesis Inc., Windham, $\mathrm{NH}$ ) and MRFA peptide in 50:50 vol/vol water/acetonitrile solution. 

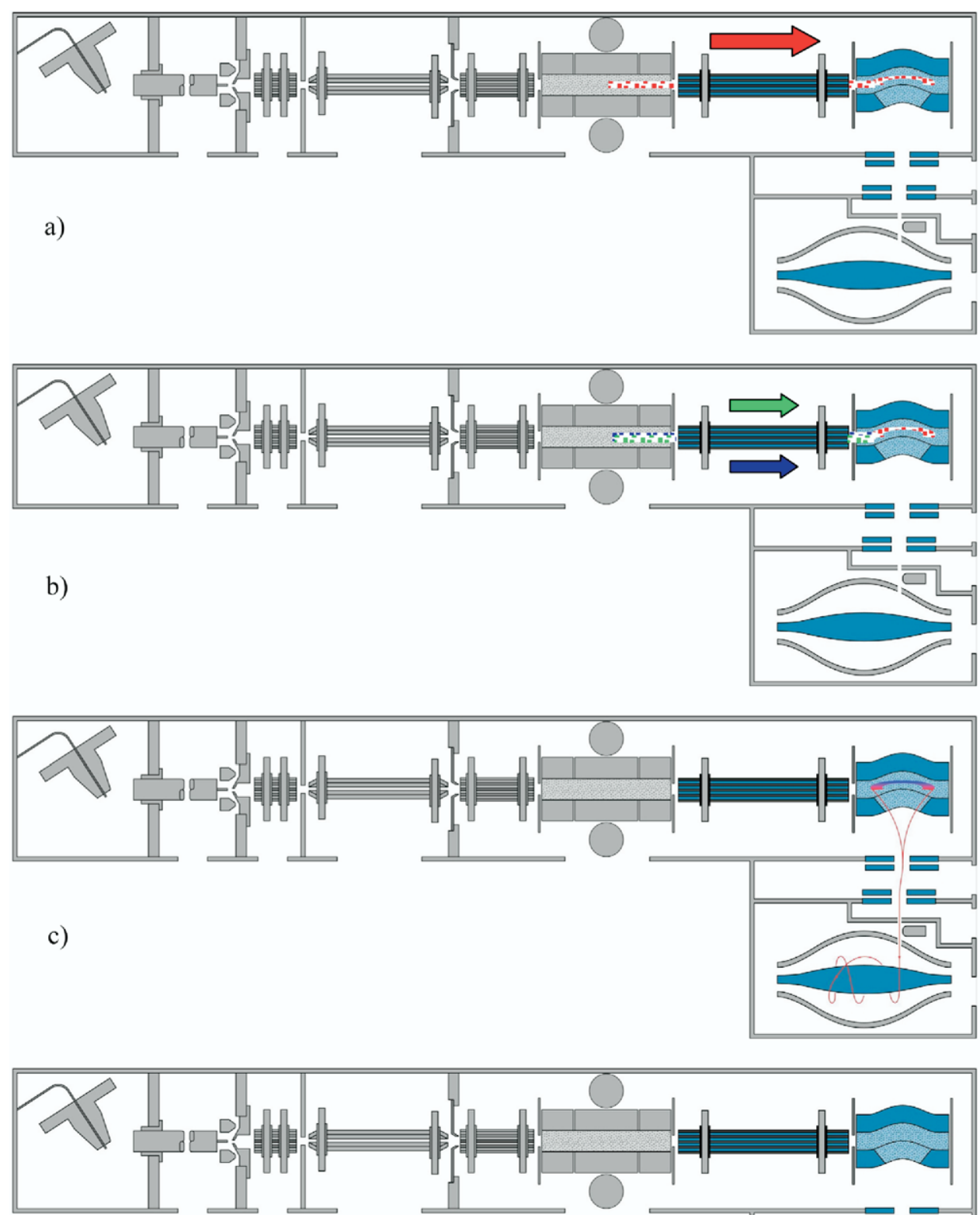

d)

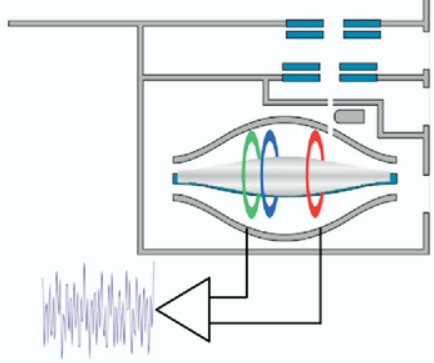

Figure 1. Experimental sequence for measurements of the extent of mass accuracy the orbitrap mass analyzer: (a) Injection of the first set of ions and trapping in the C-trap; (b) injection of the second set of ions and trapping in the C-trap; (c) pulsed injection of mixed ion population into the orbitrap; (d) ion detection in the orbitrap.

\section{Results and Discussion}

\section{Instrument Operation}

The mass spectrometer depicted in Figure 1 is a hybrid system combining a linear ion trap mass spectrometer and an orbitrap mass analyzer. Key to operation of this system is a C-shaped storage trap, which is used to store and collisionally cool ions before injection into the orbitrap. With this device, ions are pulsed into the central point of the C-trap arc that coincides with the 


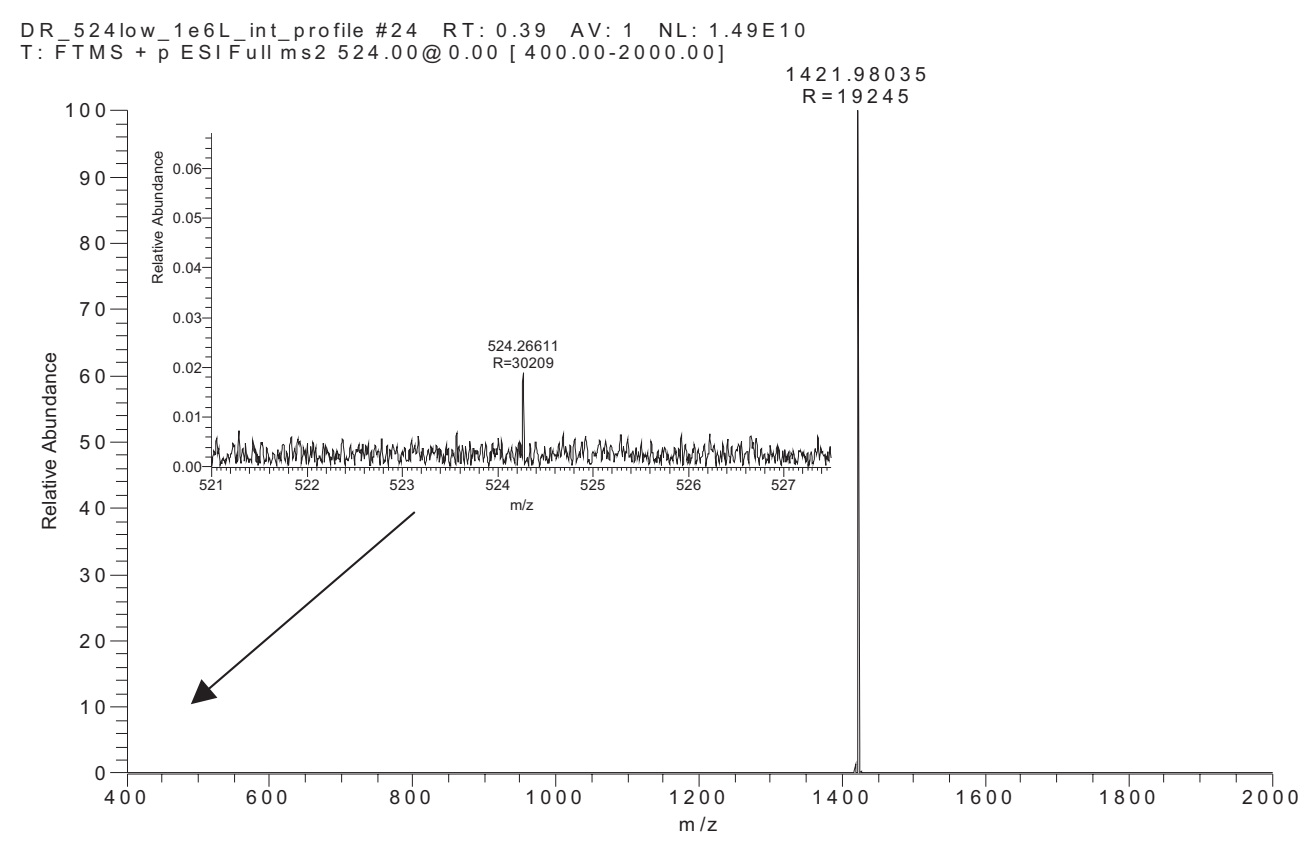

Figure 2. A typical mass spectrum acquired at the ratio 5000:1 of maximum to minimum intensities (external calibration).

orbitrap entrance aperture. Ions are captured in the orbitrap by rapidly increasing the electric field and detection of image current from coherent ion packets takes place after voltages have stabilized [16]. Signals from each of the orbitrap outer electrodes are amplified by a differential amplifier and transformed into a frequency spectrum by fast Fourier transformation. The frequency spectrum is converted into a mass spectrum using a two-point calibration and processed with Xcalibur software.

\section{Measurement Methodology}

To explore the extent of mass accuracy of the orbitrap analyzer, it is important to provide a reproducible and as wide as possible spread of signal intensities within the same spectrum. An effort was made to achieve a wide range of signal intensities by using an electrospray source with widely different concentrations of analytes. However, it appeared that competition between analytes for protons precluded the electrospray source from producing reliable and controllable signals at levels in the range 1:2000 to 1:5000, compared with the major component of the analyte mixture. To investigate properties of the orbitrap analyzer rather than the electrospray source, another approach was applied. This approach capitalized on the ability of the C-trap to store multiple fills from the linear ion trap per injection into the orbitrap, as illustrated in Figure 1. The number of ions in each fill is individually controlled over several orders of magnitude using automatic gain control (AGC), while the selection of masses in each fill is regulated using isolation in the linear ion trap. This creates a flexible and versatile tool for forming desired ion populations including, if necessary, internal calibrants. Mass calibration coefficients were determined for different AGC target values and interpolated for intermediate values. No intensity-dependant corrections of $\mathrm{m} / \mathrm{z}$ were made for data processing.

The resolving power was reduced to nominal 30,000 (at $m / z 400$ Th after zero-filling and Kaiser-Bessel apodization, $0.38 \mathrm{~s}$ transient duration) so that the experiment cycle time of $1 \mathrm{~s}$ still allowed more than sufficient time to store up to a million of ions in the C-trap. All data below correspond to a single spectrum acquisition. To model the widest possible range of conditions, intensities of dominant and minor peaks were varied over orders of magnitude to achieve variations of ratio of intensities between 1 and 10,000. For internal calibration evaluation, the intense peak was used as the calibrant. Here and below, all resolving powers are presented as full-width half-maximum (FWHM) values.

\section{Results of Measurements}

Figure 2 shows a typical mass spectrum used to determine mass errors at the extreme limits of dynamic range. Target values were $10^{6}$ for the major peak and $10^{2}$ for the minor peak; however, space charge repulsion in the C-trap resulted in a significant reduction of the major peak intensity (about 2-fold). The minor peak has such a low $\mathrm{S} / \mathrm{N}$ that noise starts to limit the precision of mass measurement in agreement with the published literature [5-7]. It this paper, noise is characterized as the maximum peak-to-peak amplitude of thermal noise of the preamplifier over the full mass range (for example, in Figure 2 the noise stays at $0.008 \%$ of the major peak). For a signal with a low $\mathrm{S} / \mathrm{N}$ ratio, it was found 
External calibration
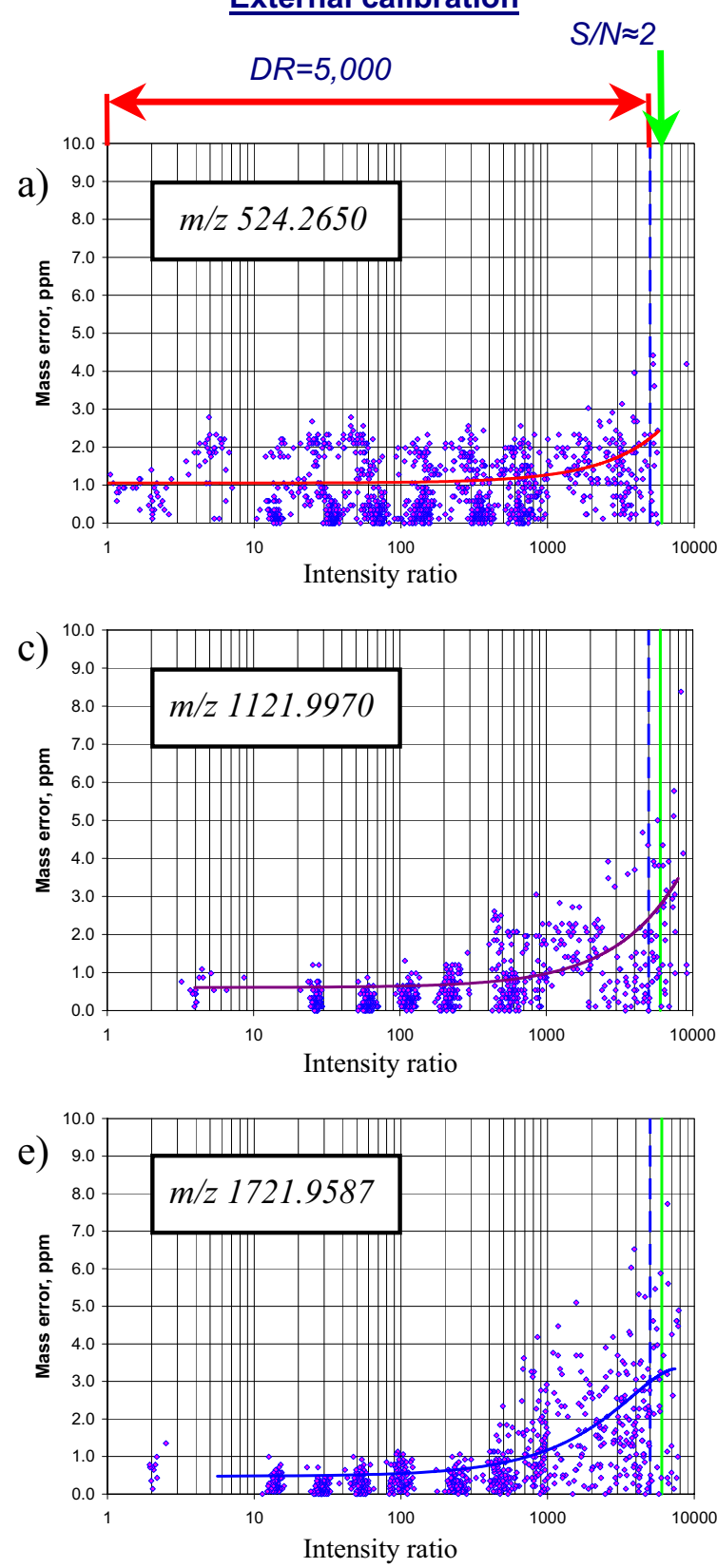

Internal calibration
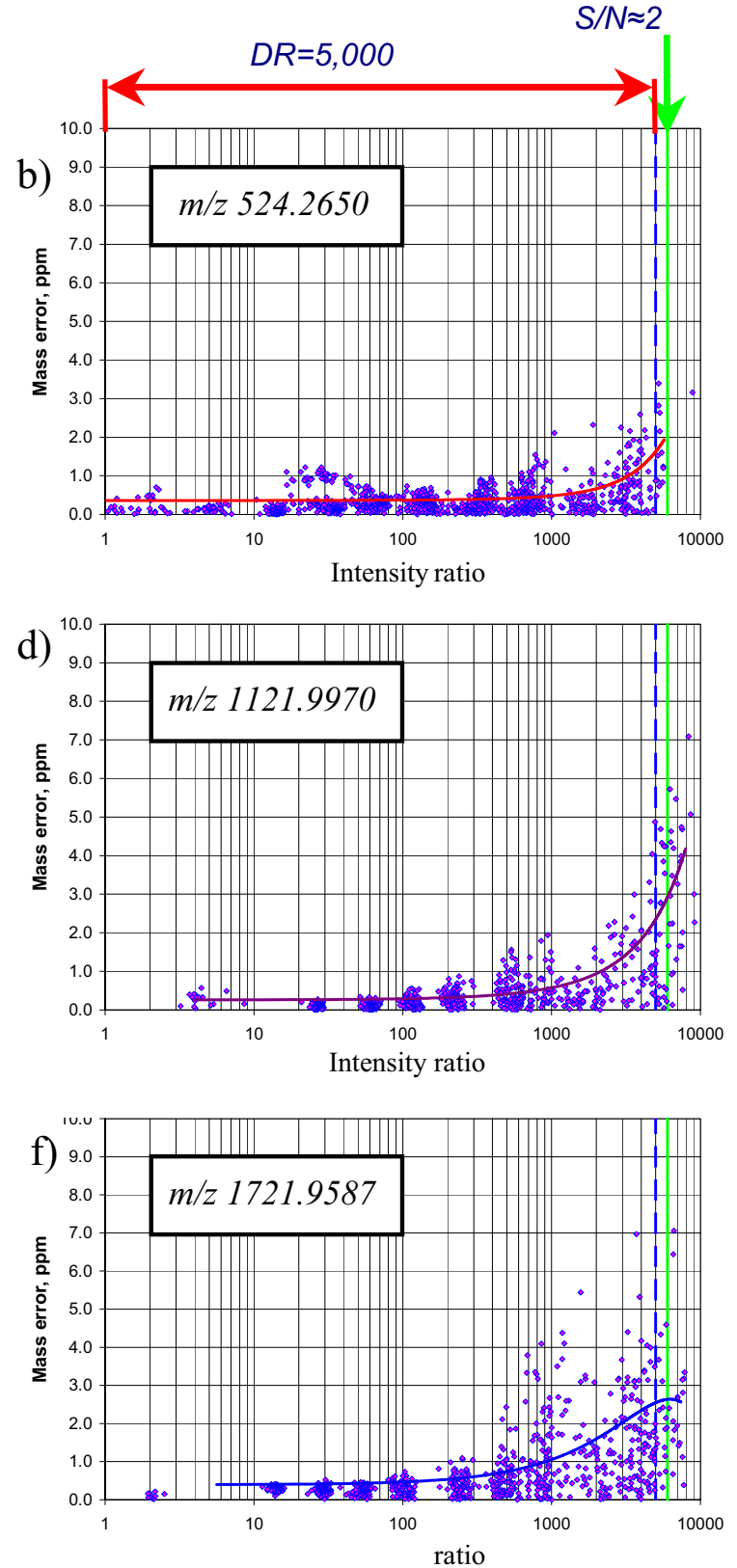

Figure 3. Absolute measured mass errors (points) and trend of r.m.s. mass error versus ratio of intensities between the major peak at $\mathrm{m} / \mathrm{z}$ 1421.97,786 (lines) and peaks at different $\mathrm{m} / \mathrm{z}:(\mathbf{a}) \mathrm{m} / \mathrm{z}=$ 524.2650 at resolving power $\mathrm{R}=30,000$, external calibration; (b) $\mathrm{m} / \mathrm{z}=524.2650$ at resolving power $\mathrm{R}$ $=30,000$, internal calibration; (c) $\mathrm{m} / \mathrm{z}=1121.9970$ at resolving power $\mathbf{R}=21,000$, external calibration; (d) $\mathrm{m} / \mathrm{z}=1121.9970$ at resolving power $\mathbf{R}=21,000$, internal calibration; (e) $\mathrm{m} / \mathrm{z}=1721.9587$ at resolving power $R=17,400$, external calibration; (f) $m / z=1721.9587$ at resolving power $R=17,400$, internal calibration.

that lower resolving power results in mass errors increasing almost proportionally. Moreover, both signal and noise increase with acquisition time, but signal increases in proportion to time, while noise increases in proportion to the square root of time. Thus, $\mathrm{S} / \mathrm{N}$ improves with acquisition time. For this reason, it is desirable to use longer acquisitions.

Figure 3 brings together numerous results of measurements for different $\mathrm{m} / \mathrm{z}$ and target values for both minor and major peaks. In all these measurements, the major peak at nominal mass 1422 was dosed with a smaller analyte with $\mathrm{m} / \mathrm{z}$ indicated in the figure along with the ratio of their signal intensities on the abscissa axis. The major peak was also used as a calibrant to produce data for internal calibration (Figure 3b, d, f). At lower values of maximum/ minimum intensity (below 100), target numbers for both major and minor peaks were varied so that the 


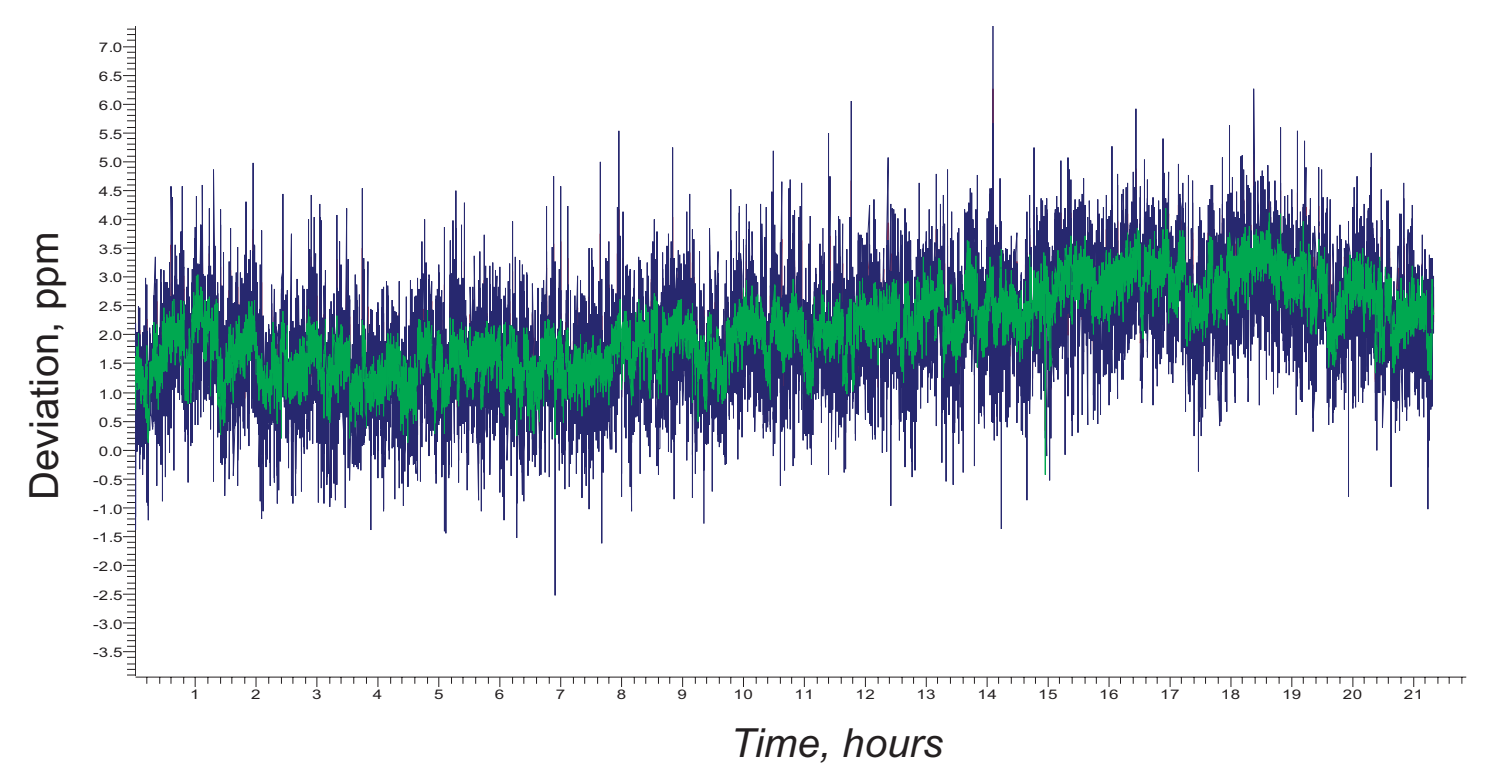

Figure 4. Long-term stability of accurate mass measurements with a large difference in signal intensities and external mass calibration (green trace: $\mathrm{m} / \mathrm{z} 1421.97,786$ at 100\%; blue trace: $\mathrm{m} / \mathrm{z}$ $524.26,496$ at $<0.02 \%)$.

total target number of stored ions was varied between $5 \cdot 10^{4}$ and $10^{6}$.

It was observed that for resolving powers of 30,000 and higher (Figure 3a, b), mass errors for all 1100 data points are under $5 \mathrm{ppm}$. For resolving powers around and below 20,000 (which are observed for higher $\mathrm{m} / \mathrm{z}$ at the same resolution setting due to a lower number of oscillations over the acquisition time), several measurements show mass errors in the range 4 to $7 \mathrm{ppm}$ (Figure $3 c-f)$. However, for all these resolving powers, rootmean-square mass errors stay well within 5 ppm down to $\mathrm{S} / \mathrm{N}_{p-p}=2$. With the major component acquired at a target number $10^{6}$, the $\mathrm{S} / \mathrm{N}_{p-p}=2$ threshold is well below $0.02 \%$ (i.e., $1: 5000$ intensity ratio); thus the extent of mass accuracy of the orbitrap analyzer extends at least up to 5000 .

At low $\mathrm{S} / \mathrm{N}$ (i.e., large $\mathrm{max} / \mathrm{min}$ intensity ratios), noise is the main contributor to the error of peak centroiding, so there is no significant difference in mass errors between internal and external calibrations. At higher $\mathrm{S} / \mathrm{N}$ ratios (small $\mathrm{max} / \mathrm{min}$ intensity ratios), precision of mass measurements improves, and for internal calibration it remains limited only by the accuracy of the two-point mass calibration. Graphs on Figure $3 \mathrm{~b}, \mathrm{~d}$, and $\mathrm{f}$ show that internal mass accuracy can be better than 1 ppm, which agrees well with published measurements for peptide mixtures [17].

For external mass accuracy, small variations of the output of the high-voltage power supply over time cause an increase of mass errors, without affecting mass errors for internal mass accuracy. This is especially pronounced for longer durations of measurements like in Figure 3a, where several acquisitions were separated by $>1 \mathrm{~h}$ from each other. The stability of external mass calibration is demonstrated in Figure
4, as shown by two mass traces with an intensity ratio of $\sim 5000$, which remained stable for more than $20 \mathrm{~h}$. In this plot, one point was acquired every $6 \mathrm{~s}$. It should be noted that mass measurement variations for the minor component is much higher because of low $\mathrm{S} / \mathrm{N}$. Spikes on the blue trace probably result from intermittent variations of minor component intensity, i.e., lower than usual $\mathrm{S} / \mathrm{N}$ ratios due to statistical effects. The overall trend demonstrates stability and effectiveness of thermal regulation, which is required for external calibration.

\section{Conclusions}

The extent of mass accuracy determines the true utility of the accurate mass capability of a mass spectrometer for real-life applications, much more than other parameters (even resolving power). From this point of view, the LTQ Orbitrap enables accurate mass measurements over an intensity range of 5000 that matches or exceeds the range of signal intensities in the electrospray ion source [18] when operated with liquid separations.

Further improvements of extent of mass accuracy can be made at both extremes of signal intensities. For low intensities, lower capacitance of the orbitrap and lower thermal noise of the image current preamplifier are needed to reduce noise and, thus, allow lower intensity signals to be detected. At high intensities, the number of injected ions appeared to saturate at levels that are still well within the space charge capacity of the orbitrap. Thus, further improvement of the C-trap would be required to approach the inherent space charge limit of the orbitrap, i.e., space charge when mass accuracy starts to drop noticeably due to Coulomb repulsion. 


\section{Acknowledgments}

The authors express their deep gratitude to colleagues from the development team of LTQ Orbitrap instrument: Wilko Balschun, Alexander Kholomeev, Dr. Kerstin Strupat, Dr. Reinhold Pesch, Frank Czemper, Oliver Hengelbrock of Thermo Electron, Bremen, Germany, and Dr. Eric Hemenway of Thermo Electron, San Jose, California, USA. The authors are also grateful to Dr. Michael Senko for his valuable comments.

\section{References}

1. Blom, K. F. Estimating the precision of exact mass measurements on an orthogonal time-of-flight mass spectrometer. Anal. Chem. 2001, 73, 715-719.

2. $\mathrm{Wu}, \mathrm{J}$.; McAllister $\mathrm{H}$. Exact mass measurement on an electrospray ionization time-of-flight mass spectrometer: Error distribution and selective averaging. J. Mass Spectrom. 2003, 38, 1043-1053.

3. Colombo, M.; Sirtori, F. R.; Rizzo, V. A fully automated method for accurate mass determination using high-performance liquid chromatography with a quadrupole/orthogonal acceleration time-of-flight mass spectrometer. Rapid Commun. Mass Spectrom. 2004, 18, 511-517.

4. Fjeldsted, J. Time-of-Flight Mass Spectrometry, Technical Overview; Report 5989-0373EN, Agilent Technologies: 2003; (http://www.chem.agilent. com)

5. Chen, L.; Cottrell, C. E.; Marshall, A. G. Effect of signal-to-noise ratio and number of data points upon precision in measurement of peak amplitude, position, and width in Fourier transform spectrometry. Chemom. Intell. Lab. Syst. 1986, 1, 51-58.

6. Verdun, F. R.; Giancaspro, C.; Marshall, A. G. Effects of noise, timedomain damping, zero-filling, and the FFT algorithm on the "exact" interpolation of fast Fourier transform spectra. Appl. Spectrosc. 1988, 42, 715-721.

7. Marshall, A. G.; Verdun, F. R. Fourier Transforms in NMR, Optical, and Mass Spectrometry; Elsevier: Amsterdam, 1990; pp 150-155.
8. Limbach, P. A.; Grosshans, P. B.; Marshall, A. G. Experimental determination of the number of trapped ions, detection limit, and dynamic range in Fourier-transform ion-cyclotron resonance mass spectrometry. Anal. Chem. 1993, 65, 135-140.

9. Easterling, M. L.; Mize, T. H.; Amster, I. J. Routine part-per-million mass accuracy for high-mass ions: Space-charge effects in MALDI FT-ICR. Anal. Chem. 1999, 71, 624-632.

10. Ledford, E. B.; Rempel, D. L.; Gross, M. L. Space-charge effects in Fourier-transform mass spectrometry-mass calibration. Anal. Chem. 1984, 56, 2744-2748.

11. Jeffries, J. B.; Barlow, S. E.; Dunn, G. H. Theory of space-charge shift of ion-cyclotron resonance frequencies. Int. J. Mass Spectrom. Ion Processes 1983, 54, 169-187.

12. Brown, C. E.; Smith, M. J. The present status and prospects for Fourier transform-ion cyclotron resonance/mass spectrometry. Spectrosc. World 1990, 1, 25-30.

13. Masselon, C.; Tolmachev, A. V.; Anderson, G. A.; Harkewicz, R.; Smith, R. D. Mass measurement errors caused by "local" frequency perturbations in FTICR mass spectrometry. J. Am. Soc. Mass Spectrom. 2002, 13, 99-106.

14. Syka, J. E. P.; Marto, J. A.; Bai, D. L.; Horning, S.; Senko, M. W.; Schwartz, J. C.; Ueberheide, B.; Garcia, B.; Busby, S.; Muratore, T.; Shabanowitz, J.; Hunt, D. F. Novel linear quadrupole ion trap/FT mass spectrometer: Performance characterization and use in the comparative analysis of histone $\mathrm{H} 3$ post-translational modifications. J. Proteome Res. 2004, 3, 621-626.

15. Schwartz, J. C.; Senko, M. W.; Syka, J. E. P. A two-dimensional quadrupole ion trap mass spectrometer. J. Am. Soc. Mass Spectrom. 2002,
13, 659-669.

16. Makarov, A. A. Electrostatic axially-harmonic orbital trapping: A novel high-performance technique of mass analysis. Anal. Chem. 2000, 72, $1156-1162$.

17. Olsen, J. V.; de Godoy, L. M.; Li, G.; Macek, B.; Mortensen, P.; Pesch, R.; Makarov, A. A.; Lange, O.; Horning, S.; Mann, M. Parts per million mass accuracy on an orbitrap mass spectrometer via lock-mass injection into a C-trap. Mol. Cell. Proteom. 2005, 4, 2010-2021.

18. Tang, K.; Page, J. S.; Smith, R. D. Charge competition and the linear dynamic range of detection in electrospray ionization mass spectrometry. J. Am. Soc. Mass Spectrom. 2004, 15, 1416-1423. 